\title{
Case study on emerging trends in geospatial technologies for study of urban form
}

\author{
Ming-Chun Lee \\ School of Architecture, University of North Carolina at Charlotte. USA \\ E-mail: Ming-Chun.Lee@uncc.edu
}

\begin{abstract}
GIS has been an effective tool to study urban form. However, as its own field, GIS has evolved in a rapid pace over the past decades. Recent developments in geospatial analytics and visualization technologies offer new tools and applications for the researchers in the field. This paper traces recent major trends in GIS and discusses their implications to the field of urban form research. These trends include the following: 1) increase in dimensions with $3 D$ GIS; 2) integration with LiDAR remote sensing; 3) cloud-based GIS; 4) integration with virtual reality. This paper identifies best practices from two recent projects in the United States. It then discusses a class project and demonstrates the potentials of these new emerging GIS technologies for the study of urban form.
\end{abstract}

Keywords: geographic information system (GIS), urban form, $3 D$ GIS, remote sensing, virtual reality.

\section{Introduction}

Geographic information system (GIS) has been used to study urban form over the past decades. It is particularly useful to measure quantitative variables of urban form, such as density, clustering, proximity, accessibility, etc (Song and Knaap, 2004; Tsai, 2005). Overall, GIS has been an effective tool for researchers in the field. However, GIS as its own field has continued to evolve in a rapid pace (Goodchild, 2011). Recent developments in the area of geospatial technologies offer new possibilities with new toolsets for spatial analysis, data visualization, and three-dimensional (3D) modeling (Kumar, 2015).

This paper traces recent major trends in GIS and discusses their implications to the field of urban form research. These trends include the following:
1) Integration with remote sensing: remote sensing not only enables $3 \mathrm{D}$ visualization with imagery processing but also provides other spatial information to create meaningful analysis results. For instance, LiDAR point cloud data allows extraction of built forms and identification of physical features and land coverages.

2) Increase in dimensions with $3 D$ GIS: conventional 2D maps are being replaced by interactive 3D models generated by procedural rules stored in GIS. Along with locations and associated attributes, vertical elevation and architectural details are also represented.

3) Cloud-based GIS: web-based GIS services allow centralized access to location-based information. Yet through distributed mobile platforms, real-time data collection, sharing, and collaboration are done seamlessly in the cloud. 
4) Integration with virtual reality: virtual reality creates immersive experiences with a perception of being physically present in a virtual world. GIS not only can greatly enhance the accuracy and realism of virtual scenes with digital elevation models and street networks, but also can assist in 3D features generation.

This paper identifies best practices from two recent projects done by two municipalities in the United States. It then discusses a class project conducted by the author and his students, and demonstrates the potentials of these new emerging GIS tools for the study of urban form.

\section{LiDAR}

LiDAR (light detection and ranging) is an optical remote sensing technology that uses laser light to densely sample the surface of the earth. It is active remote sensing. This means that LiDAR itself sends a pulse of near infrared light and it waits for the pulse to return. This is different from passive sensors, which collects reflected light from the sun. LiDAR, mainly used in airborne laser mapping applications, can produce highly accurate $\mathrm{x}-\mathrm{y}-\mathrm{z}$ measurements and mass point cloud datasets that can be managed, visualized, analyzed, and shared using GIS software programs, such as Esri's ArcGIS (Esri, 2016).

Post-processed spatially organized LiDAR data is known as point cloud data. The initial point clouds are large collections of 3D elevation points, which include $\mathrm{x}, \mathrm{y}$, and $\mathrm{z}$, along with additional attributes such as GPS time stamps. The specific surface features that the laser encounters are classified after the initial LiDAR point cloud is post-processed. Elevations for the ground, buildings, tree canopy, roadway structures, bridges, and anything else that the laser pulse encounters during the survey constitutes point cloud data (GISGeography, 2017).

Recent achievements in LiDAR technologies are changing the pace of our transition to true 3D GIS. Earlier LiDAR systems emit a pulse of laser energy and measure the time it takes for that energy to travel to a target, bounce off the target, and return to the sensor. These systems are called linear-mode because they generally only have a single aperture, and so can only measure distance along a single vector at any point in time. More recent Geiger-Mode and Photon-Counting LiDAR systems are based on focal plane-based LiDAR design. These systems are able to collect data with sampling rate of more than $200 \mathrm{MHz}$, versus the maximum $800 \mathrm{KHz}$ of the current linear-mode LiDAR, and point cloud density of up to 100 points per square meter. Such LiDAR systems collect data from an altitude up to $30,000 \mathrm{ft}$ above ground resulting in a wider ground coverage. Another important aspect that these newer LiDAR technologies offer, beside the density, is their focal plane design aspect, which results in a raster-style data acquisition and in turn produces an accurate elevation for the intended survey area. Overall, LiDAR has become the inevitable technology to provide accurate 3D data fast and reliably (Abdullah, 2015; Ullrich and Pfennigbauer, 2016).

\section{D procedural modeling}

Spatial information in 3D enables users to view and analyze data in detail and precision that cannot be achieved in 2D. 3D allows real-world views that improve the users' orientation and increase their ability to perform wide variety of tasks related to their spatial workspace. One of the recent software developments in $3 \mathrm{D}$ modeling is procedural modeling (Andrews, 2017).

Procedural modeling is an umbrella term for a number of techniques in computer graphics to create 3D models and textures from sets of rules. Procedural modeling focuses primarily on creating a model from a rule set, or enhancing models automatically. Procedural modeling is often applied when it would be too cumbersome to create a 3D model using conventional 3D modeling programs, or when more specialized tools are required. This is often the case for plants, architecture or landscapes (Muller, et al., 2006; Nishida, et al., 2016).

Esri CityEngine is a stand-alone desktop commercial application for the design, planning, and modelling of large-scale urban 
environments in $3 \mathrm{D}$. With the procedural modeling approach, CityEngine facilitates professional users in urban planning and management to quickly generate $3 \mathrm{D}$ city models from existing 2D GIS data. Urban designers can conduct conceptual design in 3D based on GIS data and procedural rules. Professionals in the filming and gaming industries can model virtual 3D urban environments for simulation, game development, and entertainment (Ribeiro, et al., 2014).

A single procedural rule can be used to generate many 3D models in CityEngine. With the integration between CityEngine and ArcGIS, a rule set can make use of feature attributes stored in GIS data, such as numbers of floors, floor heights, roof types, or wall textures, to generate different 3D models that represent properties of different features. A 3D model generated in this fashion is basically a $3 \mathrm{D}$ object resulting from a $2 \mathrm{D}$ shape extrusion according to the rules defined in a rule set, in this case a cga file. The origin of these 2D shapes can either be imported from ArcGIS or built manually in CityEngine.

CityEngine connects to ArcGIS mainly through data exchange. It now can import and export multiple file formats that are compatible with many other desktop 3D modeling applications, such as AutoCAD or SketchUp. Terrain models can be created from simple image files with georeferencing information (GeoTiff files) or from digital elevation (terrain) models (DEM or DTM). Currently, CityEngine only supports image-based terrains (gray scale height maps). It does not support 3D meshes. CityEngine provides tools to align 3D shapes or features to the terrain (Jin, et al., 2015; Ribeiro, et al., 2014).

\section{Cloud-based GIS: ArcGIS Online}

The rise of cloud computing has been one of the most significant advancements in the field of information and communication technology. Everything these days seems to be heading to the cloud. You can store your documents, images, contacts, calendars, presentations, articles, accounts, everything in the cloud. So it just seems logical that GIS is also heading that way. Most of the leading GIS vendors have developed cloud-based GIS solutions, including Esri's ArcGIS Online (Chappell, 2010).

Some of the broad advantages of cloud-based GIS include: 1) data access can be through any Internet connection, anytime, anywhere; 2) For an organization with a range of remote users, cloud-based GIS makes the distribution of GIS data, analysis and systems simple to implement and manage; 3) having a cloud GIS allows data capture in real or near real time to be displayed directly onto a centralized system.

Esri's ArcGIS Online is an online cloud-based, collaborative web GIS that allows users to use, create, and share maps, 3D scenes, applications, layers, analytics, and GIS data. Users have access to many data sources that have already been made available to the subscribers, such as Esri's Living Atlas of the World. Users can also use many templates to create their own maps or apps. They can take advantage of Esri's secure cloud, where users can add items and publish web layers or 3D scenes. ArcGIS Online is an integral part of Esri's ArcGIS system. It extends the capabilities of ArcGIS Desktop and other GIS platforms and tools (Esri, 2017).

ArcGIS Online includes a suite of basemaps that provide reference maps for the entire planet and context for local jurisdictions. These maps are built from the best available data from a community of authoritative data providers and presented in multiple cartographic styles. ArcGIS Online also includes detailed imagery of the world, which reveals both the present state of the planet and change over time. It also includes a comprehensive set of demographic and lifestyle maps of the United States through its online-based systems: Business Analyst and Community Analyst. With these ready-to-use basemaps and data, users can then add their own GIS data and create mashups that serve their own purposes (Esri, 2017).

\section{Virtual reality}

Virtual reality (VR) is an environment where the human perception of a simulated environment is as close to the perception of the real world 
as possible. VR creates immersive experiences with a perception of being physically present in a non-physical world. To experience VR, users hold a screen mounted to a headset, which is typically powered by a gaming console, a mobile phone, or a computer to their eyes. Then through specialized software and sensors, users are immersed in an artificial world where they interact and reach out to the various objects (Manly, 2015; Parisi, 2015; Rubin, 2014).

The immersive nature of VR, which allows viewers to encounter a simulated 3D landscape from multiple points of view, can be a very useful tool for urban planners, designers, or researchers in the field of urban morphology. They can use it to redraw streets and neighborhoods, offer real and imagined views of existing and proposed developments, or study historical events related to urban transformations.

With its promising potential to be a powerful tool for landscape architects, architects, planners and developers, most of them happen to be frequent GIS users, VR has already drawn attentions from the GIS industry, considered to be the next frontier to broaden the capabilities of GIS. For example, Esri has created a mobile VR solution for urban planners, architects, and GIS professionals. With their latest release of CityEngine (2016.1), users of CityEngine have the option to quickly convert their $3 \mathrm{D}$ models into VR experiences on mobile devices. Esri's ArcGIS 360 VR allows users to quickly immerse themselves (through a mobile phone with VR headset) into 3D city models by teleporting to static viewpoints and comparing different urban design scenarios. These VR experiences can be easily created in CityEngine and are hosted on ArcGIS Online
(CityEngine, 2017).

In addition to this streamlined approach offered by Esri. Users can also use commercial third-party 3D game engines (game development software programs, such as Unity or Unreal) to create VR experiences. Esri CityEngine allows users to export their creations in various formats, including .fbx files, which can then be imported into 3D game engines. Users then can add VR support to their models in these game engines (Singh, et al., 2014; Smelik, et al., 2014).

\section{Case study}

Several cities in the United States have taken advantage of these new GIS tools to create maps, 3D models and scenes, and other presentations to support their urban planning and development efforts. Some of these tools and the methods incorporated by these cities can be possibly applied to study urban design, urban form, or urban morphological processes in general.

City of Beaverton in Oregon used ArcGIS Online to create a series of 3D web scenes to study various urban planning issues, including land use, zoning, and flood risk. This was done by using the basemaps already available in ArcGIS Online to provide the context of the city, then adding City's own authoritative data to create the contents (City of Beaverton, 2016). City of Beaverton also used the LiDAR feature extraction technique to generate fairly detailed 3D building models and tree canopies. They also created a series of CityEngine models to represent a number of future development scenarios for a neighborhood in Portland as a pilot test (Figure 1).
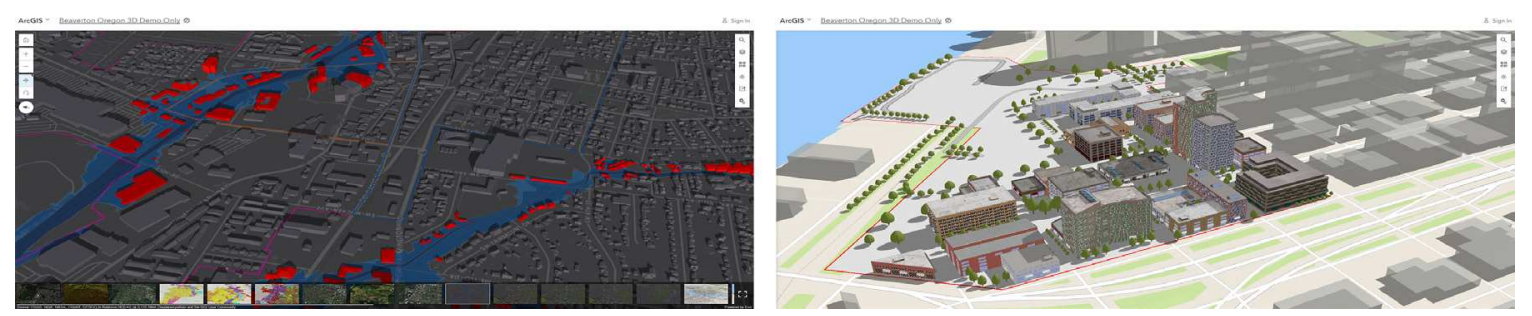

Figure 1. City of Beaverton used ArcGIS Online to create 3D web scenes to study flood risk in relation to urban form (left), and created CityEngine models to examine future development scenarios (right). 
Central Boulder in Colorado also used ArcGIS Online to create a web scene to conduct their growth factors analysis, which included existing natural physical factors about open spaces and water features, as well as man-made systems, such as bike lanes, trails, parks, etc. (Central Boulder, 2015). They also included some demographic and economic measures in the web scene, such as population, total dwelling units, land use, and housing mix. $3 \mathrm{D}$ buildings and trees were generated from LiDAR and placed on top of a built-in basemap in the web scene. To present their web scene, Central Boulder then created a story map, a web-based presentation platform designed by Esri, which allowed Central Boulder to include other forms of media in the presentation, such as photos, statistic graphs, and text narratives (Figure 2).

\section{Project introduction}

To explore the potentials of these geospatial tools and visualization media, a project was conducted by the author and his students in an urban design studio class. The Graduate Program in Urban Design in School of Architecture at the University of North Carolina at Charlotte took on the challenge to re-envision the future of an old urban renewal site in City of Atlanta, a neighborhood used to be called Buttermilk Bottom. The main idea for this academic project was to learn from City of Savannah about how the class might use those lessons to redo what was badly done three decades ago in the site in Buttermilk Bottom. The assignment was to re-design the
Buttermilk Bottom site according to the design principles observed in the plan for Savannah's historic district and their quantifiable traits.

Many GIS applications were incorporated in this urban design project, including 2D mapping (ArcGIS Desktop, ArcGIS Pro), remote sensing (Esri Local Government 3D Basemaps), 3D procedural modeling (Esri CityEngine), and cloud-based tools (ArcGIS Online).

\section{GIS data acquisitions}

To assist in both the site analysis and urban design processes, GIS was used by the class to explore physical structures in both two cities, including street networks, street block/lot configurations, open spaces, distributions of land uses, demographic data and their related socio-economic characteristics.

The GIS analysis started with collecting data. The class relied on online open-source GIS databases to acquire the needed datasets for their analyses. For the study area in Savannah, SAGIS (Savannah Area Geographic Information System) Open Data site was the primary source, which provides free access to geospatial data in a standardized format to the public. For the site in Atlanta, two sources were used by the class: DPCD (Department of Planning and Community Development) Open Data and ARC (Atlanta Regional Commission) Open Data.

A series of basemaps were constructed by combining GIS datasets in both ArcMap and ArcGIS Pro to create a digital representation of the existing urban structure for both two cities. The datasets used in these basemaps included
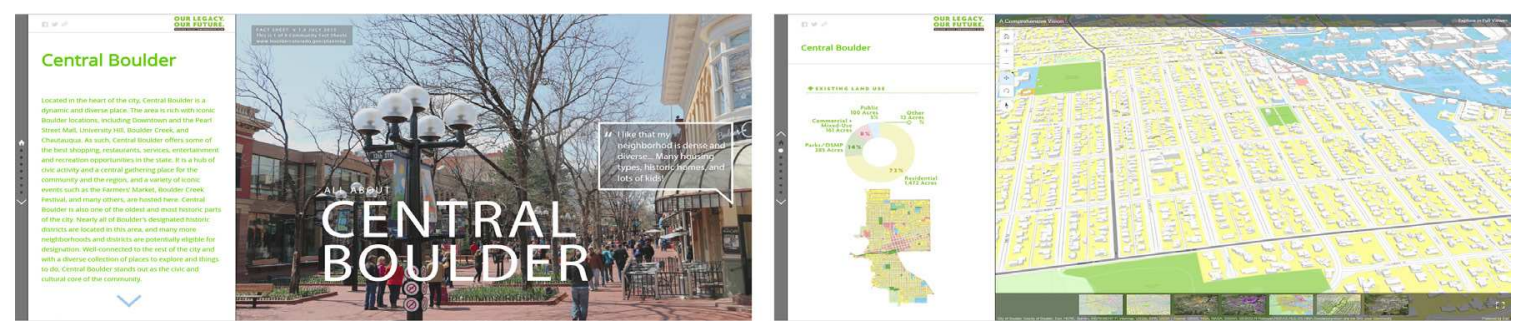

Figure 2. Central Boulder in Colorado created a story map to publicize city's images (left) and conduct growth factors analysis, such as land use in relation to building massing

(right). 
the following: streets, parcels with land use information, building footprints, parks, landmarks, points of interests (such as schools, churches), water features, contours, census tracts. Demographic data was collected from Census Bureau website (American FactFinder) and linked to the census tracts dataset.

\section{LiDAR processing}

After the basemaps being created, the next step then was to construct 3D models for both two cities. These models were intended to display the existing buildings, trees, and accurate terrains to represent the landforms. This was done by extracting 3D features from LiDAR point cloud data.

For the site in Savannah, the LiDAR data was acquired from Department of Architecture in Savannah College of Arts and Design (SCAD). Eight (8) LiDAR tiles were obtained in the las file format (version 1.1). They were commissioned in 2009, each covering an area of $5,000 \mathrm{ft}$ by $5,000 \mathrm{ft}$ with a point spacing of $1.5 \mathrm{ft}$. These tiles had been classified into 5 classes: unassigned; ground; building; noise; water.

For the site in Atlanta, the LiDAR tiles were downloaded from the website USGS Earth Explorer. Two (2) tiles were obtained in the las file format (version 1.0). The tiles were created in 2006, each covering an area of 5,300 ft by $5.300 \mathrm{ft}$ with a point spacing of $4.6 \mathrm{ft}$. Since these two LiDAR tiles were not classified, the first step was therefore to reclassify them. ArcGIS Pro was used to process this step. The reclassification was performed by applying the following two methods: classify building; classify by height.

After this step, the class used Esri's Local Government 3D Basemaps tools to process all LiDAR tiles for both two cities to extract buildings and trees in 3D (Figure 3). This step also produced digital terrain models (DTM) in the tiff file format to represent the bare ground-level terrain for both two sites.

\section{D modeling}

The 3D models created by extracting features from LiDAR data were considered simple representations of the existing urban structures for both two sites. To enhance their visual qualities and model appearances, Esri's CityEngine was used by the class to add details to the $3 \mathrm{D}$ models. These details included architectural structures and textures, landscape features, roadway signs and pavements, transportation features, vehicles and human figures. Lighting effects were also added to enhance the level of realism in the models.

Before using CityEngine, a GIS data package in the .gdb file format (Esri's File GeoDatabase) had to be prepared in ArcGIS Pro. This package included the following items: 3D buildings extracted from LiDAR by Local Government 3D Basemaps; DTM as .tiff images; tree points (also from LiDAR), streets, building footprints, aerial photos of the sites as .tiff images.

The street-creation tool in CityEngine was used to generate streets. This step was done based on street types that were identified by the class. All the details associated with the streets, such as vegetation, signs, pavements, vehicles and human figures, were added by using Esri's Complete Street rule package, which is available online. The class then used Esri's Urban Design rule package to generate 3D buildings with architectural details and textures.

Overall, the class was able to use the 3D models generated by CityEngine to examine the physical qualities of the areas, such as: overall land use distributions by color-coding building footprints; potential ways of urban transformations by urban design; streetscape configurations with fully rendered details (Figure 4).

\section{Cloud-based GIS}

In addition to relying on online open data sources to acquire GIS data, the class also took advantage of Esri's cloud-based platform, ArcGIS Online, for the following activities and purposes: 

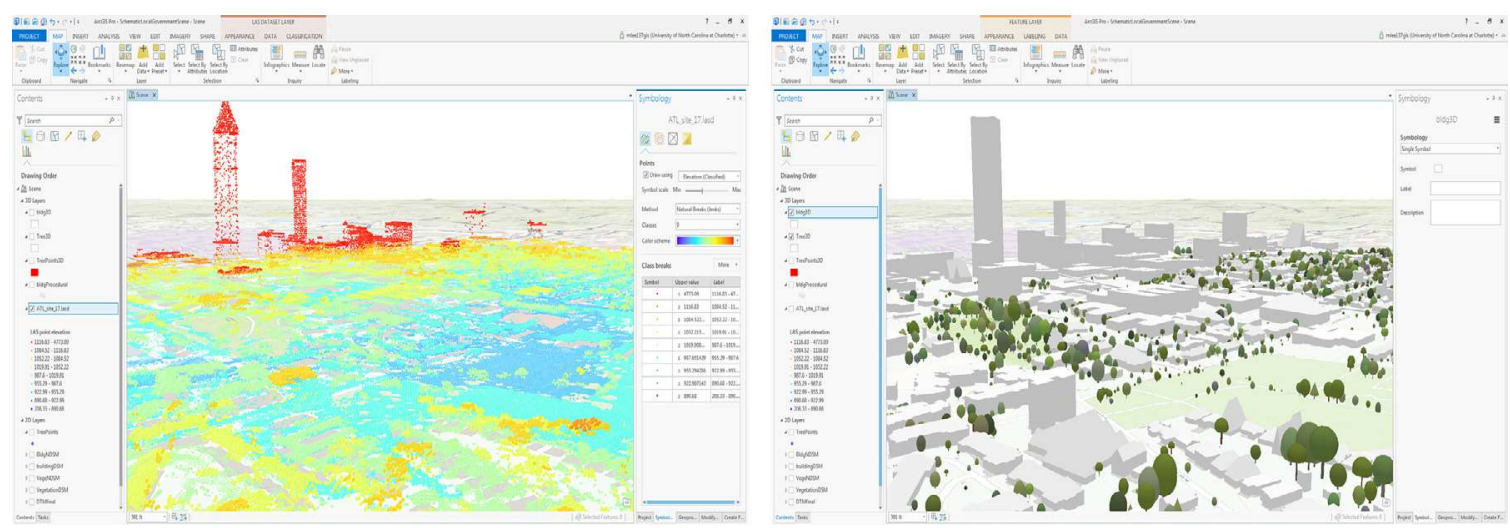

Figure 3. The class used ArcGIS Pro and followed Esri's Local Government 3D Basemaps workflow to reclassify LiDAR point cloud data (left) and extract 3D features from the LiDAR (right) for City of Atlanta.
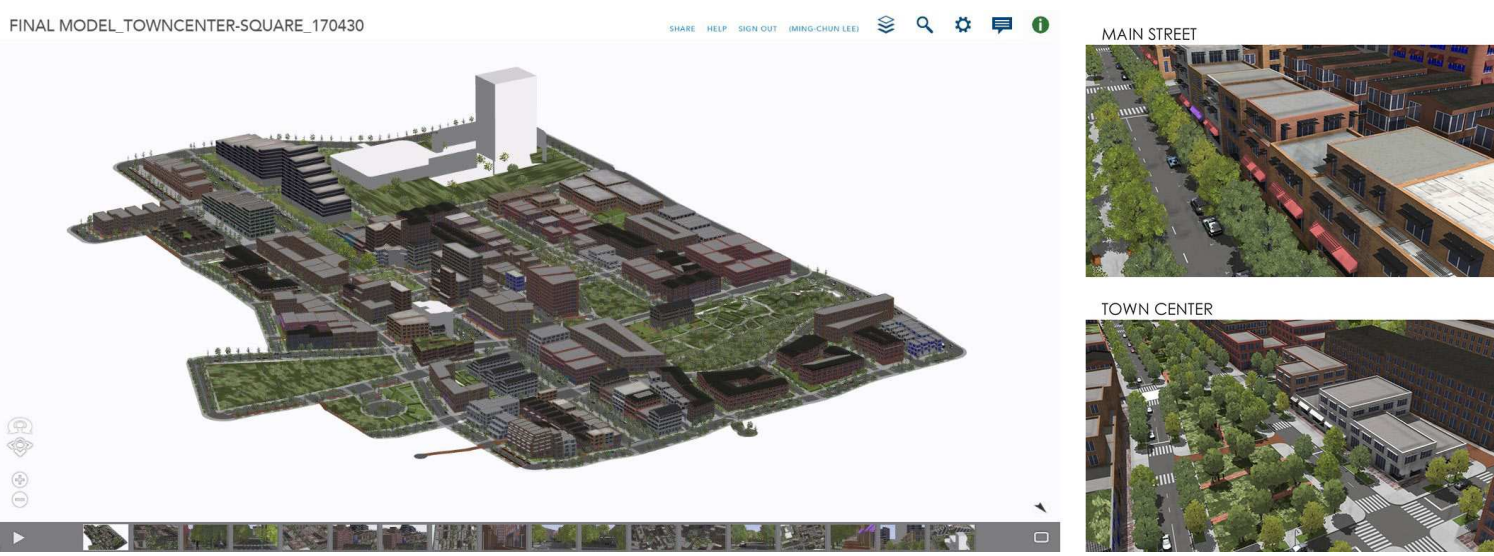

Figure 4. CityEngine allowed the class to create a large-scale model with details to render future urban design solutions.

1) Data and content sharing: An online user group was created to include all the students as well as the instructor of the class to enable sharing of GIS data, processed map layer and scene layer packages, and maps and 3D scenes that were generated by the class.

2) Data processing and analysis: the class also used some of the analysis capabilities offered by the platform to perform some basic analysis, such as walkability analysis and hot-spot analysis.

3) Map and web scene generation: the class used this online platform to generate maps and 3D web scenes, which included detailed streetscapes, buildings, and spatial analysis results, to visualize and explore urban structures and design solutions.

4) Project presentations: the class used the template provided by the online platform to create story maps to present the project outcomes. Each story map contains multiple forms of media, including static images, text narratives, interactive maps and 3D scenes that reveal urban structures of the two sites (Broyles, et al., 2017). These story maps were made available to the general public online through the Internet (Figure 5).

\section{VR development}

The class also tested the potential of using virtual reality as a way to study urban form and explore urban design solutions. Their 3D CityEngine models were exported as .fbx files (Autodesk), which were then imported into Unity, a game development engine, to be later converted into virtual reality scenes. 

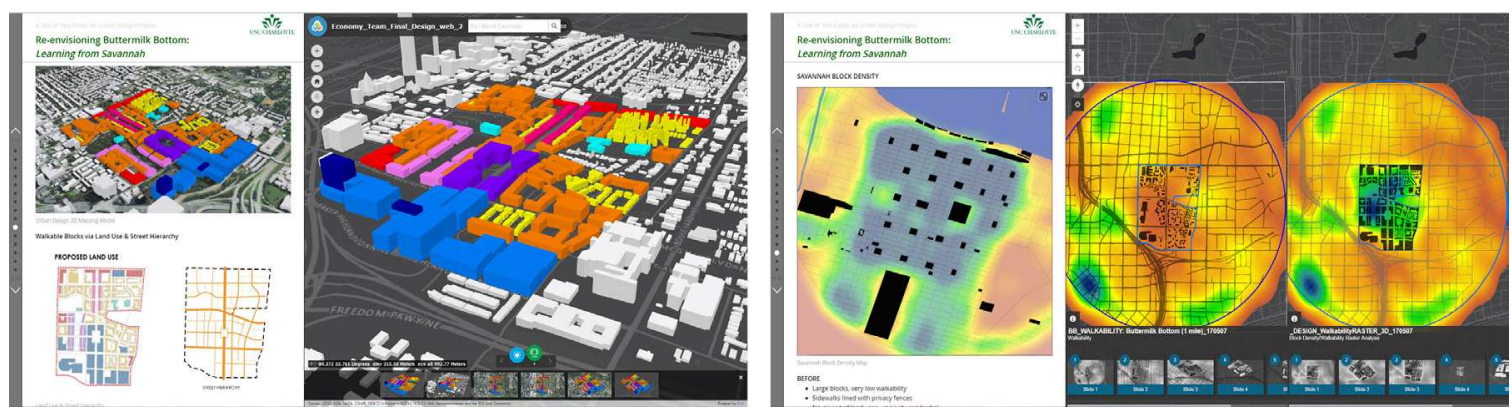

Figure 5. The class created a story map to organize a series of web scenes for documenting their analyses and design solutions, including land use mix analysis (left) and walkability analysis (right).

Unity allows additional lighting effects and environment rendering options to enhance the appearance of the models. It also enables virtual reality settings that allow a user to use a typical game controller, such as Xbox controller, to walk around 3D scenes generated by the CityEngine models. During the class final review meeting, a computer was set up to allow guest critics to experience the virtual reality scenes in person and offer comments for students' design projects (Figure 6).

\section{Discussions and conclusion}

This class exercise offered an opportunity to explore the possibilities to use new geospatial tools and visualization media to study urban form and its related topics. These tools and media, including $3 \mathrm{D}$ procedural modeling with GIS, LiDAR remote sensing, cloud-based GIS platform, and virtual reality, represent four of the many major development trends in the geospatial technology industry and its related fields.

This final section presents some of the author's initial thoughts about the potential advantages of these tools for the study of urban form, as well as some noticeable disadvantages or issues that may face prospect users.

\section{Advantages:}

1) Quick 3D form extraction from LiDAR: With the recent advancements in the LiDAR technologies and the lower costs of deployment and data acquisitions nowadays, as well as with some significant efforts from several federal agencies in the United States to establish geospatial databases accessible to the public, it has become quite possible to obtain high quality LiDAR data at no cost within the States. Furthermore, modern GIS software packages have also become more powerful in their abilities to leverage LiDAR data for point clouds reclassification, ground terrain identification, and 3D feature extraction. For this project, LiDAR data processing offered a convenient way to study the existing physical fabric of the two study sites by revealing their landforms and quickly extracting built structures from the LiDAR data.

2) Urban structure exploration with $3 D$ procedural modeling: Procedural modeling with its rule-based approach to 3D form generation has made it possible to quickly create detailed $3 \mathrm{D}$ city models for a large geographic area. This has become possible in particular with the integration between GIS and the procedural modeling program itself. Now users can import GIS data into the modeling program and use the attributes that are already embedded in the data, such as land use classifications or building heights, then link certain attributes with an appropriate set of form-generating rules to build 3D models. Users can then explore different urban form configurations, such as street width, distance between building facades and street edges, or even tree canopy or vegetation coverage, by simply changing certain rules associated with the intended physical features. The students in the class used CityEngine's Complete Street rule package to create a series of street typologies by simply changing parameters associated with street configurations, such as the number of lanes, availability of public transit, or vegetation on sidewalks. They also 

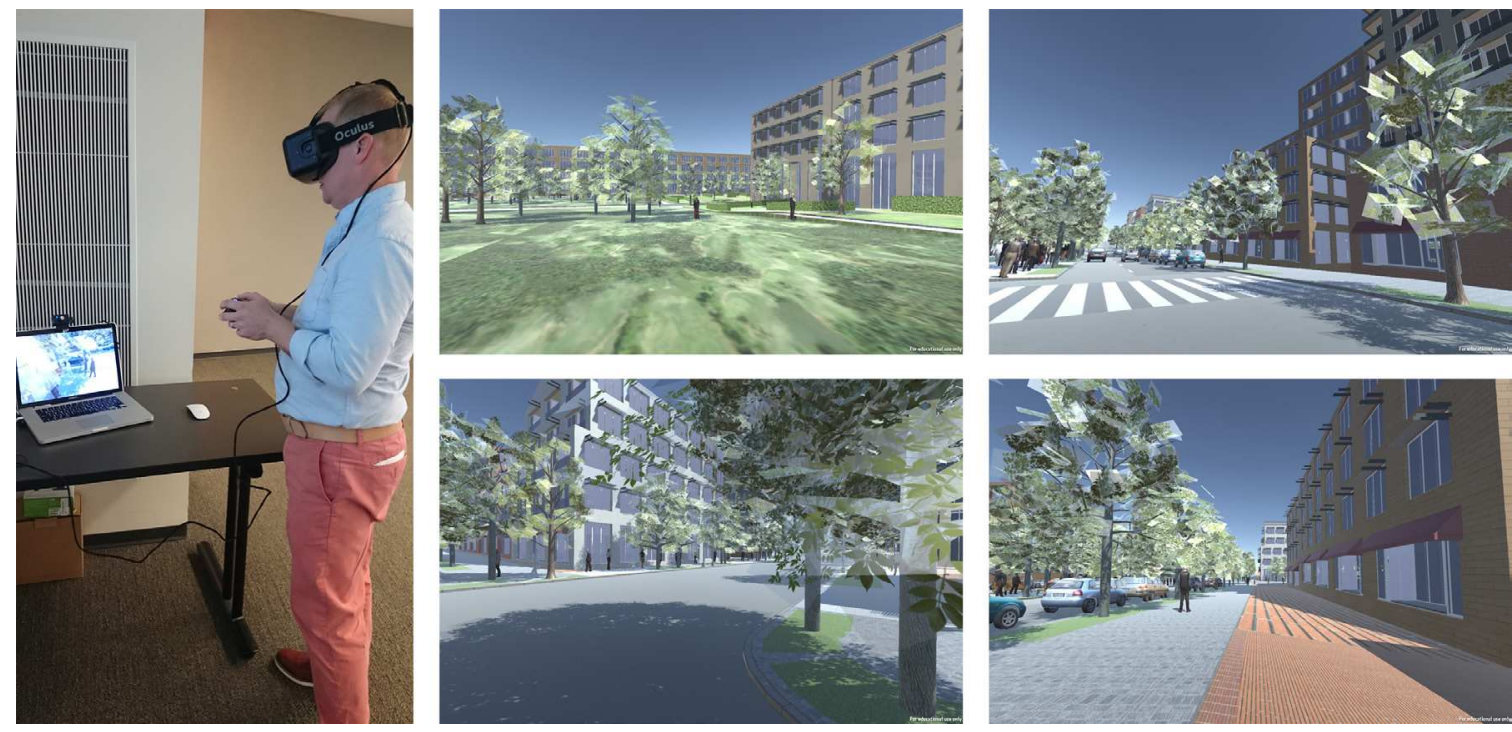

Figure 6. Virtual reality scenes, converted from CityEngine models, allowed reviewers to experience students' urban design solutions in an immersive way.

imported building footprints GIS data into CityEngine and created 3D massing models based on the land use information that came with the GIS data.

3) Easy access to accurate terrain elevation data and global satellite imagery: The cloud-based ArcGIS Online platform provides ready-to-use basemaps with accurate terrain data and satellite imagery. The class created many web maps and 3D scenes by combining their own data and 3D models with the basemaps already available in ArcGIS Online. With the elevation information and the imagery showing the ground features in the sites, the students were able to verify the accuracy of their data and models and further examine the spatial structures in the sites.

4) Easy data sharing and presentation over the Internet through cloud-based platform: The cloud-based platform also enables easy data sharing and project presentation over the Internet. The class shared their map layer packages with one another as a group in ArcGIS Online. By doing so, they were able to include some of the contents that were made by other teammates in their own maps or 3D scenes for presentation purposes. The class also created story maps using Esri's templates to document their projects. All these story maps are accessible online to the public.

5) New way to experience urban spaces through virtual reality: VR creates immersive experiences with a perception of being physically present in a non-physical world. GIS can greatly enhance the accuracy and realism of virtual scenes with up-to-date terrain models and geographically referenced 3D features. The class combined multiple software programs to take 2D GIS data, turn them into 3D models, and eventually walk around these models in a VR environment. This has been made possible through the interoperability across many different programs, including desktop GIS, procedural modeling tool, and game development engine.

\section{Disadvantages and issues}

1) Proprietary software license: All the computer programs used in the class are proprietary software. They are not free of charge. The class was able to access all these programs at no cost with institutional or educator licenses. However, the students will lose this privilege of free access once they graduate from the school.

2) Data availability and quality: Although public GIS open data is becoming more and more accessible nowadays, still not all the data are being made available to the public. Even if some data are available, they may be incomplete or out of date. The LiDAR data used for the study area in Atlanta was created in 2006 with relatively lower resolution. 
3) Inconsistent data schema: The attributes that are included in GIS data are not standardized across different jurisdictions. Different cities may have their own protocols of coding GIS data and setting up attributes. For example, land use information may be classified very differently from one city to another.

4) Multiple software programs and platforms with steep learning curve: There is just no one-button solution that would magically make things happen. This has already been true for most of the conventional GIS software programs, such as market-dominating ArcGIS. One can just imagine the complication when you need to include other programs, each of which requires intensive training and learning before it can be deployed.

5) High cost of VR devices: Most of the VR technologies are still in development. Newer devices and programs are yet to come to the market. Those options already available to the consumers are in general very pricy. Although inexpensive devices are coming, such as Google Cardboard or Samsung Gear VR, they tend to suffer from lower graphic quality and limited support in terms of visual effects.

\section{Final thought}

Though an in-depth evaluation on the exact effectiveness of these software tools or media for the study of urban form is out of scope for this paper, which can certainly be an important next-step for advancing this particular practice, this project allowed for a quick glimpse of the on-going development trends in the GIS world. As these digital tools are becoming more and more advanced, we researchers, scholars, or designers interested in understanding the dynamics of city building processes should explore new methods and establish new workflows to utilize these tools in order to advance our work.

\section{References}

Abdullah, Q. (2015) 'The Emerging Signs of 3D GIS', LiDAR News Magazine, Vol. 5, No. 4.

Andrews, C. (2017) '3D at Esri: 2016 in Review', ArcGIS Blog (https://blogs.esri. com/esri/arcgis/2017/01/04/3d-at-esri- 2016-in-review/)

Broyles, C., Huan, Y., Prioleau, B., Young, V. and Lee, M.-C. (2017) Re-envisioning Buttermilk Bottom: Learning from Savannah (http://unccharlotte.maps.arcgis.com/apps/ MapJournal/index.html?appid $=6 \mathrm{fe} 203 \mathrm{~d} 7 \mathrm{f}$ 08749dcae454136b50e1f48), accessed 15 June 2017.

Central Boulder (2015) All about Central Boulder (http://esrigeodesign.maps.arcgis. com/apps/MapJournal/index.html?appid $=492 \mathrm{~b} 1426 \mathrm{fd} 2248 \mathrm{fabb} 49 \mathrm{ec} 15 \mathrm{cbb} 4 \mathrm{c} 547$ ), accessed 15 June 2017.

Chappell, D. (2010) GIS in the Cloud: the Esri Example (http://www.esri.com/library/ whitepapers/pdfs/gis-in-the-cloud-chappell. pdf), accessed 23 May 2017.

City of Beaverton (2016) Beaverton Oregon 3D Demo Only (https://www.arcgis.com/home/ webscene/viewer.html? webscene $=7 \mathrm{f} 11$ be 6 d62ef4298bfla4bb60de66f05), accessed 15 June 2017.

CityEngine Help (2017) Introduction: The 360 VR Experience (3VR) (http://cehelp.esri. $\mathrm{com} / \mathrm{help} /$ index.jsp?topic $=/$ com.procedural. cityengine.help/html/manual/export/vr360/ export_formats_3vr.html), accessed 23 May 2017.

Esri (2016) What is LiDAR data? ArcGIS Help 10.1 (http://resources. arcgis.com/en/help/main/10.1/index. html\#//015w00000041000000), accessed 23 May 2017.

Esri (2017) What is ArcGIS Online? ArcGIS Online Help (http://doc.arcgis.com/en/ arcgis-online/reference/what-is-agol.htm), accessed 23 May 2017.

GISGeography (2017) A Complete Guide to LiDAR: Light Detection and Ranging, (http://gisgeography.com/lidar-lightdetection-and-ranging/), accessed 23 May 2017.

Goodchild, M.F. (2011) 'Looking Forward: Five Thoughts on the Future of GIS', ArcWatch (http://www.esri.com/news/ arcwatch/0211/future-of-gis.html)

Jin, X., Wang, F., Hao, L., Duan, Y., and Chen, L. (2015) 'Analysis of the Modeling Method and Application of 3D City Model based on the CityEngine', International Conference on Advances in Mechanical Engineering 
and Industrial Informatics (AMEII 2015), pp. 34-39.

Kumar, M. (2015) 'Evolution of the Geospatial Industry: From GIS to Spatial Computing', GeoAwesomeness (http://geoawesomeness. com/evolution-of-the-geospatial-industryfrom-gis-to-spatial-computing/)

Manly, L. (2015) 'A Virtual Reality Revolution, Coming to a Headset Near You', The New York Times, November 19, 2015.

Muller, P., Wonka, P., Haegler, S., Ulmer, A., and Van Gool, L. (2006) 'Procedural modeling of buildings', ACM Transactions on Graphics (TOG), Vol. 25 (3), pp. 614623.

Nishida, G., Garcia-Dorado, I., Aliaga, D. G., Benes, B., and Bousseau, A. (2016) 'Interactive Sketching of Urban Procedural Models', ACM Transactions on Graphics (TOG) - Proceedings of ACM SIGGRAPH 2016, Vol. 35 (4).

Parisi, T. (2015) Learning Virtual Reality: Developing Immersive Experiences and Applications for Desktop, Web, and Mobile, O'Reilly Media, Incorporated, 2015

Ribeiro, A., Duarte de Almeida, J.-P. and Ellul, C. (2014) 'Exploring CityEngine as a Visualization Tool for 3D Cadastre', 4th International Workshop on FIG 3D Cadastres, 9-11 November 2014, Dubai, United Arab Emirates, pp. 197-218.

Rubin, P. (2014) 'The Inside Story Of Oculus Rift And How Virtual Reality Became Reality', Wired Magazine, May 2014.

Singh, S. P., Jain, K., and Mandla, V. R. (2014) 'Image-based Virtual 3D Campus modeling by using CityEngine', American Journal of Engineering Science and Technology Research, Vol. 2, No. 1, pp. 1-10.

Smelik, R. M., Tutenel, T., Bidarra, R., and Benes, B. (2014) 'A survey on procedural modelling for virtual worlds', Computer Graphics Forum, Vol. 33, pp. 31-50.

Song, Y. and Knaap, G.-J. (2004) 'Measuring Urban Form: Is Portland Winning the War on Sprawl?', Journal of the American Planning Association, Vol. 70, No. 2, pp.210-225.

Tsai, Y.-H. (2005) 'Quantifying Urban Form: Compactness versus Sprawl', Urban Studies, Vol. 42, No. 1, pp.141-161.

Ullrich, A. and Pfennigbauer, M. (2016) 'Linear
LIDAR versus Geiger-mode LIDAR: Impact on data properties and data quality', Laser Radar Technology and Applications XXI, Proc. of SPIE, v. 9832. 